\title{
Antiagregantes y anticoagulantes: lo que el endoscopista debe saber
}

\author{
Xochiquétzal Sánchez-Chávez* \\ Hospital San Angel Inn Patriotismo, Ciudad de México, México
}

\begin{abstract}
Resumen
El uso de terapias antitrombóticas ha aumentado de manera significativa, incrementando con esto, el riesgo de hemorragia. Entre las causas y sitios de hemorragia asociada a estas terapias están aquellas que suceden alrededor de procedimientos invasivos como la endoscopia. Durante la Semana Europea de Gastroenterología (UEG) 2018 celebrada en Viena y la Semana de Enfermedades Digestivas (DDW2019) en la ciudad de San Diego, se presentaron un total de 27 trabajos relacionados con el tema de antiagregantes y anticoagulantes y endoscopia.
\end{abstract}

Palabras clave: Antiagregantes. Anticoagulantes. Endoscopia.

\section{Introducción}

El uso de terapias antitrombóticas ha aumentado progresivamente, incrementando el riesgo de hemorragia. Algunos autores han sugerido que debido al corto tiempo con el que se suspenden estos medicamentos antes de un procedimiento, el riesgo de hemorragia y trombosis puede estar subestimado.

La terapia antitrombótica incluye tanto los antiagregantes como a los anticoagulantes. En los pacientes recibiendo terapia antitrombótica y que serán sometidos a algún procedimiento invasivo como la endoscopia gastrointestinal, se debe equilibrar adecuadamente el riesgo de hemorragia frente al riesgo trombótico (arterial o venoso). Esto se debe valorar de manera individual para minimizar el tiempo en que el paciente estará sin la debida protección antiplaquetaria o anticoagulante.

Para brindar el mayor beneficio a los pacientes en la toma de decisiones, es necesario conocer los diferentes fármacos disponibles, sus indicaciones y vida media. El manejo de la terapia antitrombótica durante una endoscopia se ha hecho más complejo debido al desarrollo y prescripción de anticoagulantes de acción directa (AAD), cada uno de los cuales cuenta con potencia y vida media distinta'.

Durante la semana Europea de Gastroenterología (UEG) 2018 celebrada en Viena y la semana de enfermedades digestivas (DDW) 2019 en la ciudad de San Diego, se presentaron un total de 27 trabajos, 3 y 24 , respectivamente, relacionados con el tema de antiagregantes, anticoagulantes y endoscopia, de los cuales 12 fueron presentados de manera oral y 15 en forma de póster.

Ali Sara, et al., presentaron de manera oral, Interrupción de la anticoagulación para la gastrostomía endoscópica percutánea, ¿Es necesaria? Como es sabido, la gastrostomía endoscópica percutánea (GEP) es considerada un procedimiento de alto riesgo para hemorragia. La anticoagulación con warfarina 0 anticoagulantes de acción directa (AAD) pueden incrementar

\section{Correspondencia:}


el riesgo de hemorragia durante o después del procedimiento. La interrupción temporal de los anticoagulantes orales 0 una terapia puente se sugiere para procedimientos de alto riesgo, poniendo en balance el riesgo tromboembólico. El objetivo del estudio fue investigar la incidencia de la hemorragia en pacientes sometidos a una GEP y los que se les interrumpió la terapia con warfarina o AAD. Los datos se recolectaron de manera retrospectiva de aquellas GEP que se realizaron entre enero del 2010 y mayo del 2016, en un centro de tercer nivel. Se analizaron un total de 1710 GEP, de estos se realizó interrupción de warfarina o AAD en $138(8.1 \%)$ relacionado a alto riesgo tromboembólico. La warfarina se interrumpió en 108 (78.3\%) procedimientos y $20(21.7 \%)$ con interrupción de los AAD. No hubo datos de hemorragia en aquellos pacientes en los que se interrumpió warfarina ni AAD. Este estudio demuestra que la interrupción temporal de anticoagulantes o la terapia puente en pacientes con alto riesgo tromboembólico no es necesaria si se realiza en un hospital con múltiples procedimientos ${ }^{2}$.

Fujinami Haruka, et al. presentaron en forma de cartel, las tendencias recientes en la detección de la hemorragia y factores de riesgo de sangrado gastrointestinal en pacientes que reciben terapia con anticoagulantes. Este estudio informa que en la última década, la causa principal de hemorragia secundaria a una úlcera péptica es el uso de antitrombóticos y AINE, siendo anteriormente la infección por Helicobacter pylori. Con los avances en el tratamiento en la terapia cardiovascular, la terapia antiplaquetaria dual (TAD) y los anticoagulantes de acción directa (AAD) se utilizan cada vez más. Existe una fuerte evidencia que la hemorragia gastrointestinal $(\mathrm{HG})$ superior se ha reducido por la combinación de un inhibidor de la bomba de protones (IBP) con la aspirina y la terapia antiplaquetaria. El objetivo del estudio, fue evaluar a 24,183 pacientes ambulatorios que se sometieron a endoscopia superior (ES), cápsula endoscópica (CE) y enteroscopia asistida con dispositivos (EAD) en el hospital universitario de Toyama, Japón entre enero del 2010 y diciembre del 2017. Los criterios de inclusión fueron: 1) pacientes con $\mathrm{HG}$ evidente, 2) lesiones sangrantes del estómago y duodeno en la ES, y 3) hemorragia de intestino delgado (HID) diagnostica por CE o EAD. Se recolecto el sitio de la hemorragia y el uso de terapia antitrombótica (aspirina como monoterapia, TAD, warfarina y AAD) de los expedientes de los pacientes. Cumplieron con los criterios de inclusión 165. Se mostró que las tasas de HG superior se disminuyeron y las tasas HID se incrementaron. El modelo de regresión logística múltiple para la HID, el uso de la TAD (RM igual 2.89; IC 95\% = 2.34-3-42; $p=0.048$ ), warfarina (RM 2.89; IC 95\% = 2.90-3.99; $p=0.024$ ) y AAD $(\mathrm{RM}=23.57$; IC $95 \%=22.50-24.64 ; \mathrm{p}=0.003) \mathrm{se}$ asociaron significativamente con un alto riesgo de HID. Concluyendo que la HID se ha incrementado y esto se relaciona con el uso de los antitrombóticos ${ }^{3}$.

Fukunaga Shusei, et al. presentaron en cartel, La puntación de ORBIT predice mayor riesgo de hemorragia después de polipectomía colorrectal en pacientes que están recibiendo terapia con anticoagulantes orales. El objetivo del estudio fue investigar el uso del puntaje de ORBIT en el riesgo de hemorragia pospolipectomía en colon (RHPPC). Fue un estudio retrospectivo, en un solo centro de estudio, realizado entre enero del 2013 y 2018, se realizaron polipectomías en 1362 colonoscopias con 5,106 lesiones. De estos se incluyeron 121 pacientes que tomaban terapia antitrombótica con 157 lesiones, incluyendo 36 pacientes que fueron sometidos a polipectomías en días diferentes, si alguno tenía varios pólipos se tomaba en cuenta la lesión más grande. El puntaje de ORBIT se calculó del total del puntaje de los siguientes factores: reducción de la $\mathrm{Hb} / \mathrm{H}$ to e historia de hemorragia (2 puntos cada uno); insuficiencia renal crónica, tratamiento antiplaquetario y >74 años (1 punto cada uno). Aquellos pacientes que tenían $<2$ puntos se consideraban de bajo riesgo, y aquellos que tenían $>3$ puntos se consideraban de alto riesgo. Ellos concluyeron que el puntaje de ORBIT puede ser útil para predecir el RHPPC principalmente en pacientes que reciben tratamiento con anticoagulación oral ${ }^{4}$.

\section{Conflicto de intereses}

Speaker de Ferring S.A. DE C.V y de Medtronic, México. Sin patrocinio de la industria.

\section{Bibliografía}

1. Peláez-Luna Mario, Blanco-Vela Claudia Isabel, et al. Guía de manejo de terapia antitrombótica durante procedimientos endoscópicos. Endoscopia. 2019:31:58-76.

2. Ali Sara, Kannadatha Bijun S, et al. Safety profile of percutaneous endoscopic gastrostomy (PEG) in Geriatric Patients: A retrospective Study. Poster Mo1341. DDW2019.

3. Fujinami Haruka, Mihara Hiroshi, et al. Recent trends in bleeding location and risk factors for gastroitnestinal bleeding in patients receiving anticoagulation therapy. Poster Mo1285. DDW2019.

4. Fukunaga S. et al. Orbit Score predicts major bleeding after colorectal polipectomy in patient receiving oral anticoagulation therapy. Poster Su1690. DDW 2019. 